\title{
POSITIVE MINIMAL REALIZATION OF CONTINUOUS-DISCRETE LINEAR SYSTEMS WITH ALL-POLE AND ALL-ZERO TRANSFER FUNCTION
}

\author{
Łukasz SAJEWSKI \\ ${ }^{*}$ Faculty of Electrical Engineering, Białystok University of Technology, ul. Wiejska 45D, 15-351 Białystok, Poland \\ l.sajewski@pb.edu.pl
}

\begin{abstract}
The positive and minimal realization problem for continuous-discrete linear single-input and single-outputs (SISO) systems is formulated. Two special case of the continuous-discrete systems are given. Method based on the state variable diagram for finding a positive and minimal realization of a given proper transfer function is proposed. Sufficient conditions for the existence of a positive minimal realization of a given proper transfer function of all-pole and all-zero systems are established. Two procedures for computation of a positive minimal realization are proposed and illustrated by a numerical examples.
\end{abstract}

Key words: Continuous-Discrete, 2D, Minimal, Positive, Realization, Existence, Computation

\section{INTRODUCTION}

In positive systems inputs, state variables and outputs take only non-negative values. Examples of positive systems are industrial processes involving chemical reactors, heat exchangers and distillation columns, storage systems, compartmental systems, water and atmospheric pollution models. A variety of models having positive linear systems behavior can be found in engineering, management science, economics, social sciences, biology and medicine, etc. Positive linear systems are defined on cones and not on linear spaces. Therefore, the theory of positive systems is more complicated and less advanced. An overview of state of art in positive systems theory is given in the monographs: Farina and Rinaldi (2000), Kaczorek (2002). The realization problem for positive discrete-time and continuous-time systems without and with delays was considered in Kaczorek and Busłowicz (2004), Kaczorek (2004, 2005, 2006a, 2006b).

Continuous-discrete 2D linear system is a dynamic system that incorporates both continuous-time and discrete-time dynamics. It means that state vector of $2 \mathrm{D}$ system contain continuoustime state variables and discrete-time state variables, input and output vectors depends on continuous time $t$ and discrete steps $i$. Examples of continuous-discrete systems include systems with relays, switches, and hysteresis, transmissions, and other motion controllers, constrained robotic systems, automated highway systems, flight control and management systems, analog/digital circuit. The positive continuous-discrete $2 \mathrm{D}$ linear systems have been introduced in Kaczorek (2002), positive hybrid linear systems in Kaczorek (2007) and the positive fractional 2D hybrid systems in Kaczorek (2008a). Different methods of solvability of $2 \mathrm{D}$ hybrid linear systems have been discussed in Kaczorek et al. (2008) and the solution to singular 2D hybrids linear systems has been derived in Sajewski (2009). The realization problem for positive 2D hybrid systems have been addressed in Kaczorek (2002, 2008b), Sajewski and Kaczorek (2009, 2010) and the minimal realization problem for all-pole (the transfer function with only poles) and all-zero (the transfer function with all zero poles) 2D systems has been addressed in Antoniou (2002) and Varoufakis et al. (1987).

The main purpose of this paper is to present a method for computation of a positive and minimal realization of a given proper transfer function of all-pole and all-zero continuousdiscrete linear system. Proposed method is based on the state variable diagram method. Sufficient conditions for the existence of a positive minimal realization of a given proper transfer function of all-pole and all-zero system will be established and a procedures for computation of a positive minimal realization for two cases of transfer functions will be proposed. The paper is organized as follows. In section 2 some preliminaries concerning the positive continuous-discrete 2D linear systems and minimal realization are recalled and the positive minimal realization is formulated. Two special cases of continuous-discrete systems are recalled in section 3 . In the same section the solution to the positive minimal realization problem for two cases of transfer function are presented and the sufficient conditions for existence of positive minimal realization are derived. Concluding remarks are given in section 4 .

To the best author knowledge the positive minimal realization problem for continuous-discrete $2 \mathrm{D}$ linear systems have not been considered yet.

In the paper the following notation will be used. The set of $n \times m$ real matrices will be denoted by $\mathfrak{R}^{n \times m}$ and $\mathfrak{R}^{n}=\mathfrak{R}^{n \times 1}$. The set of $n \times m$ real matrices with nonnegative entries will be denoted by $\mathfrak{R}_{+}^{n \times m}$ and $\mathfrak{R}_{+}^{n}=\mathfrak{R}_{+}^{n \times 1} . M_{n}$ be the set of $n \times m$ Metzler matrices (real matrices with nonnegative offdiagonal entries). The $n \times n$ identity matrix will be denoted by $I_{n}$ and the transpose will be denoted by $T$.

\section{PRELIMINARIES AND PROBLEM FORMULATION}

Consider a continuous-discrete linear system described by the equations (Kaczorek, 2002):

$\dot{x}_{1}(t, i)=A_{11} x_{1}(t, i)+A_{12} x_{2}(t, i)+B_{1} u(t, i)$ 


$$
\begin{aligned}
& t \in \mathfrak{R}_{+}=[0,+\infty] \\
& x_{2}(t, i+1)=A_{21} x_{1}(t, i)+A_{22} x_{2}(t, i)+B_{2} u(t, i) \\
& i \in Z_{+} \\
& y(t, i)=C_{1} x_{1}(t, i)+C_{2} x_{2}(t, i)+D u(t, i)
\end{aligned}
$$

where: $\quad \dot{x}_{1}(t, i)=\frac{\partial x_{1}(t, i)}{\partial t}, \quad x_{1}(t, i) \in \mathfrak{R}^{n_{1}}, \quad x_{2}(t, i) \in \mathfrak{R}^{n_{2}}$, $u(t, i) \in \mathfrak{R}^{m}, y(t, i) \in \mathfrak{R}^{p}$ and $A_{11} \in M_{n_{1}}, A_{12} \in \mathfrak{R}^{n_{1} \times n_{2}}$, $A_{21} \in \Re^{n_{2} \times n_{1}}, A_{22} \in \mathfrak{R}^{n_{2} \times n_{2}}, B_{1} \in \mathfrak{R}^{n_{1} \times m}, B_{2} \in \mathfrak{R}^{n_{2} \times m}$, $C_{1} \in \mathfrak{R}^{p \times n_{1}}, C_{2} \in \mathfrak{R}^{p \times n_{2}}, D \in \mathfrak{R}^{p \times m}$ are real matrices.

Boundary conditions for (1a) and (1b) have the form:

$$
x_{1}(0, i)=x_{1}(i), i \in Z_{+} \text {and } x_{2}(t, 0)=x_{2}(t), t \in \mathfrak{R}_{+}
$$

Note that the continuous-discrete linear system (1) has a similar structure as the Roesser model (Kaczorek, 2007; Roesser, 1975).

Definition 2.1. The continuous-discrete linear system (1) is called internally positive if $\mathrm{x}_{1}(t, i) \in \mathfrak{R}_{+}^{n_{1}}, \quad x_{2}(t, i) \in \mathfrak{R}_{+}^{n_{2}}$ and $y(t, i) \in \mathfrak{R}_{+}^{p}, t \in \mathfrak{R}_{+}, i \in Z_{+}$for all arbitrary boundary conditions $x_{1}(i) \in \mathfrak{R}_{+}^{n_{1}}, i \in Z_{+}, x_{2}(t) \in \mathfrak{R}_{+}^{n_{2}}, t \in \Re_{+}$and all inputs $u(t, i) \in \mathfrak{R}_{+}^{m}, t \in \mathfrak{R}_{+}, i \in Z_{+}$.

Theorem 2.1. (Kaczorek, 2002; 2007) The continuous-discrete linear system (1) is internally positive if and only if:

$$
\begin{aligned}
& A_{11} \in M_{n_{1}}, A_{12} \in \mathfrak{R}_{+}^{n_{1} \times n_{2}}, A_{21} \in \mathfrak{R}_{+}^{n_{2} \times n_{1}}, A_{22} \in \mathfrak{R}_{+}^{n_{2} \times n_{2}}, \\
& B_{1} \in \mathfrak{R}_{+}^{n_{1} \times m}, B_{2} \in \mathfrak{R}_{+}^{n_{2} \times m}, C_{1} \in \mathfrak{R}_{+}^{p \times n_{1}}, C_{2} \in \mathfrak{R}_{+}^{p \times n_{2}}, \\
& D \in \mathfrak{R}_{+}^{p \times m} .
\end{aligned}
$$

The transfer matrix of the system (1) is given by the formula:

$$
\begin{aligned}
T(s, z) & =\left[\begin{array}{ll}
C_{1} & C_{2}
\end{array}\right]\left[\begin{array}{cc}
I_{n_{1}} s-A_{11} & -A_{12} \\
-A_{21} & I_{n_{2}} z-A_{22}
\end{array}\right]^{-1}\left[\begin{array}{l}
\bar{B}_{1} \\
\bar{B}_{2}
\end{array}\right] \\
& +D \in \mathfrak{R}^{p \times m}(s, z)
\end{aligned}
$$

where $\mathfrak{R}^{p \times m}(s, z)$ is the set of $p \times m$ real matrices in $s$ and $z$ with real coefficient. Considering the $m$-inputs and $p$-outputs continuous-discrete linear system (1), the proper transfer matrix will be having the following form:

$$
T(s, z)=\left[\begin{array}{ccc}
T_{11}(s, z) & \ldots & T_{1 m}(s, z) \\
\vdots & \vdots & \vdots \\
T_{p 1}(s, z) & \ldots & T_{p m}(s, z)
\end{array}\right] \in \mathfrak{R}^{p \times m}(s, z)
$$

where:

$$
T_{k l}(s, z)=\frac{Y_{k l}(s, z)}{U_{k l}(s, z)}=\frac{\sum_{i=0}^{n_{k l}} \sum_{j=0}^{m_{k l}} b_{i, j}^{k l} s^{i} z^{j}}{s^{n_{k l}} z^{m_{k l}}-\left(\sum_{\substack{i=0 \\ i+j \neq n_{k l}+m_{k l}}}^{n_{k l}} \sum_{j=0}^{m_{k l}} a_{i, j}^{k l} s^{i} z^{j}\right)}
$$

For $k=1,2, \ldots, p ; \quad l=1,2, \ldots, m$ where $U(s, z)=$ $Z\{L[u(t, i)]\}, y(s, z)=Z\{L[y(t, i)]\}$ and $Z$ and $L$ are the zet and Laplace operators.

Multiplying the numerator and denominator of transfer matrix (5b) by $s^{-n_{1, k l}} Z^{-n_{2, k l}}$ we obtain the transfer matrix in the state space form eg. form which is needed to draw the state space diagram (Kaczorek, 1992; Roesser, 1975; Sajewski and Kaczorek, 2010):

$$
T_{k l}\left(s^{-1}, z^{-1}\right)=\frac{\sum_{i=0}^{n_{1, k l}} \sum_{j=0}^{n_{2, k l}} b_{i, j}^{k l} s^{-i} z^{-j}}{s^{-n_{1, k l}} z^{-n_{2, k l}}-\left(\sum_{\substack{i=0 \\ i+j \neq n_{1, k l}+n_{2, k l}}}^{n_{1, k l} n_{2, k l}} a_{i, j}^{k l} s^{-i} z^{-j}\right)}
$$

for $k=1,2, \ldots, p ; l=1,2, \ldots, m$.

Definition 2.2. The matrices (3) are called the positive realization of the transfer matrix $T(s, z)$ if they satisfy the equality (4). The realization is minimal if the matrix $A$ have lowest possible dimension among all realizations. For given transfer matrix there exist many sets of matrices $A, B C, D$ but for given matrices $A, B C$, $D$ there exist only one transfer function.

The positive minimal realization problem can be stated as follow.

Given a proper rational matrix $T(s, z) \in \mathfrak{R}^{p \times m}(s, z)$, find its positive and minimal realization (3).

Taking under considerations Definition 2.2 and e.g. similarity transformation $[8,9]$ the solution to the realization problem given in Section 3 is not unique.

Remark 2.1. For $1 \mathrm{D}$ systems the minimal realization is the one with the matrix $A$ of dimension $n \times n$ where $\mathrm{n}$ is the degree of the characteristic polynomial of the system (Kaczorek, 1992). This was implicated by controllability and observability of the 1D system. For 2D system in general case this relationship is not true (Sun-Yuan et al., 1977) and observability connected with controllability of the 2D system does not implicate the minimality of its realization.

Remark 2.2. The minimal realization for $2 \mathrm{D}$ system is the one with the matrix $A$ of dimension $\left(n_{1}+n_{2}\right) \times\left(n_{1}+n_{2}\right)$ where $n_{1}$ and $n_{2}$ are the degrees of the characteristic polynomial in $s$ and $z$ of the system (Sun-Yuan et al., 1977).

\section{PROBLEM SOLUTION FOR SISO SYSTEMS}

The solution to the minimal positive realization problem will be presented on two special cases of the $2 \mathrm{D}$ transfer functions ( $m=p=1)$. Proposed method will be based on state variable diagram (Kaczorek, 2002; Sajewski and Kaczorek, 2010). Lets consider the following two cases of the transfer functions of continuous-discrete linear system.

Case 1. The transfer function of all-pole system (which is the transfer function with only poles):

$$
T\left(s^{-1}, z^{-1}\right)=\frac{b}{1-\sum_{\substack{i=0 \\ i+j \neq 0}}^{n_{1}} \sum_{\substack{j=0 \\ i}}^{n_{2}} a_{i, j} s^{-i} z^{-j}}=\frac{Y}{U}
$$

where $b$ is the real coefficient.

Case 2. The transfer function of all-zero system (which is the transfer function with all zero poles):

$T\left(s^{-1}, z^{-1}\right)=\frac{\sum_{i=0}^{n_{1}} \sum_{j=0}^{n_{2}} b_{i, j} s^{-i} z^{-j}}{1}=\frac{Y}{U}$ 


\subsection{Case 1}

Defining:

$$
E=\frac{U}{1-a_{n_{1}, n_{2}-1} z^{-1}-a_{n_{1}-1, n_{2}} s^{-1}-\ldots-a_{00} s^{-n_{1}} z^{-n_{2}}}
$$

from (7) we obtain:

$$
\begin{aligned}
& E=U+\left(a_{n_{1}, n_{2}-1} z^{-1}+a_{n_{1}-1, n_{2}} s^{-1}+\ldots+a_{00} s^{-n_{1}} z^{-n_{2}}\right) E, \\
& Y=b E .
\end{aligned}
$$

Using (10) we may draw the state variable diagram shown on Fig. 1.

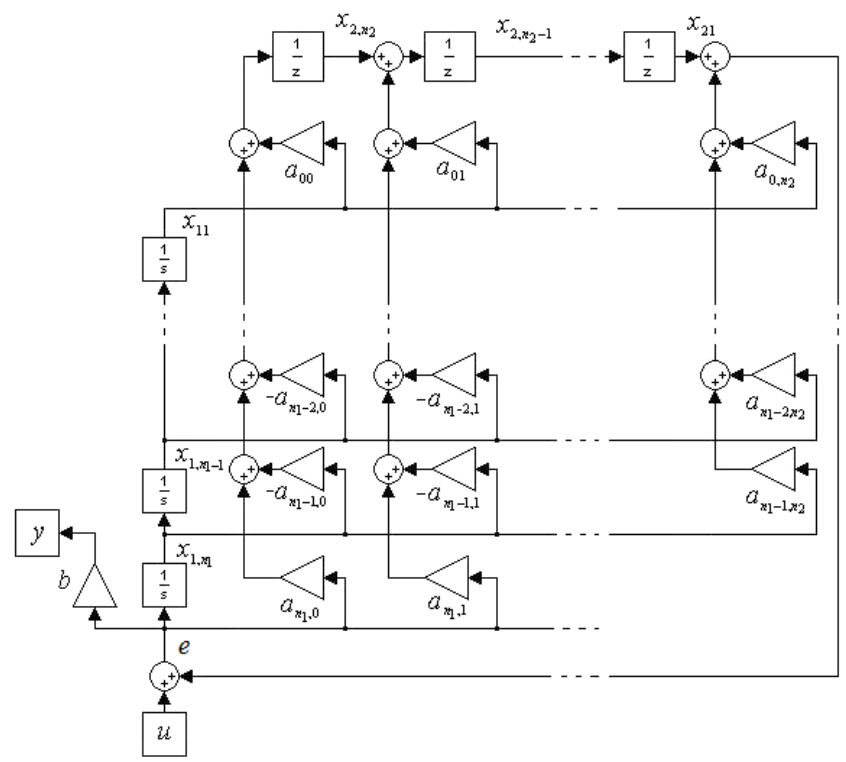

Fig. 1. State variable diagram for transfer function (7) of all-pole system

As a state variable we choose the outputs of integrators $\left(\mathrm{x}_{1,1}(t, i), \mathrm{x}_{1,2}(t, i), \ldots, \mathrm{x}_{1, \mathrm{n}_{1}}(t, i)\right)$ and of delay elements $\left(\left(\mathrm{x}_{2,1}(t, i), \mathrm{x}_{2,2}(t, i), \ldots, \mathrm{x}_{2, \mathrm{n}_{2}}(t, i)\right)\right.$. Using state variable diagram (Fig. 1) we can write the following differential and difference equations:

$$
\begin{aligned}
& \dot{x}_{1,1}(t, i)=x_{1,2}(t, i), \\
& \dot{x}_{1,2}(t, i)=x_{1,3}(t, i), \\
& \vdots \\
& \dot{x}_{1, n_{1}-1}(t, i)=x_{1, n_{1}}(t, i), \\
& \dot{x}_{1, n_{1}}(t, i)=e(t, i), \\
& x_{2,1}(t, i+1)=a_{0, n_{2}-1} x_{1,1}(t, i)+a_{1, n_{2}-1} x_{1,2}(t, i)+\ldots \\
& \quad+a_{n_{1}-1, n_{2}-1} x_{1, n_{1}}(t, i)+x_{2,2}(t, i)+a_{n_{1}, n_{2}-1} e(t, i), \\
& x_{2,2}(t, i+1)=a_{0, n_{2}-2} x_{1,1}(t, i)+a_{1, n_{2}-2} x_{1,2}(t, i)+\ldots \\
& \quad+a_{n_{1}-1, n_{2}-2} x_{1, n_{1}}(t, i)+x_{2,3}(t, i)+a_{n_{1}, n_{2}-2} e(t, i), \\
& \quad \vdots \quad \\
& \quad x_{2, n_{2}-1}(t, i+1)=a_{0,1} x_{1,1}(t, i)+a_{1,1} x_{1,2}(t, i)+\ldots \\
& \quad+a_{n_{1}-1,1} x_{1, n_{1}}(t, i)+x_{2, n_{2}}(t, i)+a_{n_{1}, 1} e(t, i),
\end{aligned}
$$

$$
\begin{aligned}
& x_{2, n_{2}}(t, i+1)=a_{0,0} x_{1,1}(t, i)+a_{1,0} x_{1,2}(t, i)+\ldots \\
& \quad+a_{n_{1}-1,0} x_{1, n_{1}}(t, i)+a_{n_{1}, 0} e(t, i), \\
& y(t, i)=b e(t, i),
\end{aligned}
$$

where:

$$
\begin{gathered}
e(t, i)=a_{0, n_{2}} x_{1,1}(t, i)+a_{1, n_{2}} x_{1,2}(t, i)+\ldots \\
+a_{n_{1}-1, n_{2}} x_{1, n_{1}}(t, i)+x_{2,1}(t, i)+u(t, i) .
\end{gathered}
$$

Substituting (11b) into (11a) we obtain:

$$
\begin{aligned}
& \dot{x}_{1,1}(t, i)=x_{1,2}(t, i), \\
& \dot{x}_{1,2}(t, i)=x_{1,3}(t, i) \text {, } \\
& \dot{x}_{1, n_{1}-1}(t, i)=x_{1, n_{1}}(t, i) \text {, } \\
& \dot{x}_{1, n_{1}}(t, i)=a_{0, n_{2}} x_{1,1}(t, i)+a_{1, n_{2}} x_{1,2}(t, i)+\ldots \\
& +a_{n_{1}-1, n_{2}} x_{1, n_{1}}(t, i)+x_{2,1}(t, i)+u(t, i), \\
& x_{2,1}(t, i+1)=\bar{a}_{0, n_{2}-1} x_{1,1}(t, i)+\bar{a}_{1, n_{2}-1} x_{1,2}(t, i) \\
& +a_{n_{1}, n_{2}-1} u(t, i)+\ldots+\bar{a}_{n_{1}-1, n_{2}-1} x_{1, n_{1}}(t, i) \\
& +a_{n_{1}, n_{2}-1} x_{2,1}(t, i)+x_{2,2}(t, i), \\
& \text { : } \\
& x_{2, m-1}(t, i+1)=\bar{a}_{0,1} x_{1,1}(t, i)+\bar{a}_{1,1} x_{1,2}(t, i)+\ldots \\
& +\bar{a}_{n_{1}-1,1} x_{1, n_{1}}(t, i)+a_{n_{1}, 1} x_{2,1}(t, i)+x_{2, m}(t, i)+a_{n_{1}, 1} u(t, i), \\
& x_{2, m}(t, i+1)=\bar{a}_{0,0} x_{1,1}(t, i)+\bar{a}_{1,0} x_{1,2}(t, i)+\ldots \\
& +\bar{a}_{n_{1}-1,0} x_{1, n_{1}}(t, i)+a_{n_{1}, 0} x_{2,1}(t, i)+a_{n_{1}, 0} u(t, i), \\
& y(t, i)=b a_{0, n_{2}} x_{1,1}(t, i)+b a_{1, n_{2}} x_{1,2}(t, i)+\ldots \\
& +b a_{n_{1}-1, n_{2}} x_{1, n_{1}}(t, i)+b x_{2,1}(t, i)+b u(t, i),
\end{aligned}
$$

where:

$\bar{a}_{i, j}=a_{i, j}+a_{i, n_{2}} a_{n_{1}, j}$

for $i=0,1, \ldots, n_{1}-1, j=0,1, \ldots, n_{2}-1$

Defining state vectors in the form:

$$
x_{1}(t, i)=\left[\begin{array}{c}
x_{1,1}(t, i) \\
\vdots \\
x_{1, n_{1}}(t, i)
\end{array}\right], \quad x_{2}(t, i)=\left[\begin{array}{c}
x_{2,1}(t, i) \\
\vdots \\
x_{2, n_{2}}(t, i)
\end{array}\right]
$$

we can write the equations (12) in the form:

$$
\begin{aligned}
& {\left[\begin{array}{c}
\dot{x}_{1}(t, i) \\
x_{2}(t, i+1)
\end{array}\right]=\left[\begin{array}{ll}
A_{11} & A_{12} \\
A_{21} & A_{22}
\end{array}\right]\left[\begin{array}{l}
x_{1}(t, i) \\
x_{2}(t, i)
\end{array}\right]+\left[\begin{array}{c}
B_{1} \\
B_{2}
\end{array}\right] u(t, i),} \\
& y(t, i)=\left[\begin{array}{ll}
C_{1} & C_{2}
\end{array}\right]\left[\begin{array}{l}
x_{1}(t, i) \\
x_{2}(t, i)
\end{array}\right]+D u(t, i),
\end{aligned}
$$

where:

$$
A_{11}=\left[\begin{array}{ccccc}
0 & 1 & 0 & \ldots & 0 \\
0 & 0 & 1 & \ldots & 0 \\
\vdots & \vdots & \vdots & \ddots & \vdots \\
0 & 0 & 0 & \ldots & 1 \\
a_{0, n_{2}} & a_{1, n_{2}} & a_{2, n_{2}} & \ldots & a_{n_{1}-1, n_{2}}
\end{array}\right] \in \mathfrak{R}^{n_{1} \times n_{1}},
$$




$$
\begin{aligned}
A_{12} & =\left[\begin{array}{cccc}
0 & 0 & \ldots & 0 \\
\vdots & \vdots & \ldots & \vdots \\
0 & 0 & \ldots & 0 \\
1 & 0 & \ldots & 0
\end{array}\right] \in \mathfrak{R}^{n_{1} \times n_{2}}, \\
A_{21} & =\left[\begin{array}{ccc}
\bar{a}_{0, n_{2}-1} & \ldots & \bar{a}_{n_{1}-1, n_{2}-1} \\
\vdots & \ldots & \vdots \\
\bar{a}_{0,0} & \ldots & \bar{a}_{n_{1}-1,0}
\end{array}\right] \in \mathfrak{R}^{n_{2} \times n_{1}}, \\
A_{22} & =\left[\begin{array}{ccccc}
a_{n_{1}, n_{2}-1} & 1 & 0 & \ldots & 0 \\
a_{n_{1}, n_{2}-2} & 0 & 1 & \ldots & 0 \\
\vdots & \vdots & \vdots & \ddots & \vdots \\
a_{n_{1}, 1} & 0 & 0 & \ldots & 1 \\
a_{n_{1}, 0} & 0 & 0 & \ldots & 0
\end{array}\right] \in \mathfrak{R}^{n_{2} \times n_{2}} \\
B_{1} & =\left[\begin{array}{c}
0 \\
\vdots \\
0 \\
1
\end{array}\right] \in \mathfrak{R}^{n_{1} \times 1}, B_{2}=\left[\begin{array}{c}
a_{n_{1}}, n_{2}-1 \\
a_{n_{1}}, n_{2}-2 \\
\vdots \\
a_{n_{1}, 0}
\end{array}\right] \in \mathfrak{R}^{n_{2} \times 1}, \\
C_{1} & =\left[\begin{array}{lll}
b a_{0, n_{2}} & \ldots & b a_{n_{1}-1, n_{2}}
\end{array}\right] \in \mathfrak{R}^{1 \times n_{1}}, \\
C_{2} & =\left[\begin{array}{lll}
b & \ldots & 0
\end{array}\right] \in \mathfrak{R}^{1 \times n_{2}}, \quad D=[b] \in \mathfrak{R}^{1 \times 1} .
\end{aligned}
$$

Therefore, the following theorem has been proved.

Theorem 3.1. There exists a positive realization of dimension $\left(n_{1}+n_{2}\right) \times\left(n_{1}+n_{2}\right)$ if the system is all-pole and all coefficients of the nominator and denominator of the transfer function (7) are nonnegative.

If the assumptions of Theorem 3.1 are satisfied then a positive realization (3) of (7) can be found by the use of the following procedure.

\section{Procedure 3.1}

Step 1. Write the transfer function (7) in the form (10).

Step 2. Using (10) draw the state variable diagram shown in Fig. 1.

Step 3. Choose the state variables and write equations (12).

Step 4. Using (12) find the desired realization (15) of transfer function (7).

Example 3.1. Find a positive realization (3) of the all-pole continuous-discrete system with proper transfer function:

$$
T\left(s^{-1}, z^{-1}\right)=\frac{2}{1-0.5 z^{-1}-0.4 s^{-1}-0.3 s^{-1} z^{-1}-0.2 s^{-2}-0.1 s^{-2} z^{-1}} .
$$

In this case $n_{1}=2$ and $n_{2}=1$.

Using Procedure 3.1 we obtain the following.

Step 1. Transfer function (16) can be written as:

$$
\begin{aligned}
& E=U+\left(0.5 z^{-1}+0.4 s^{-1}+0.3 s^{-1} z^{-1}+0.2 s^{-2}+0.1 s^{-2} z^{-1}\right) E, \\
& Y=2 E .
\end{aligned}
$$

Step 2. State variable diagram has the form shown on Fig. 2.

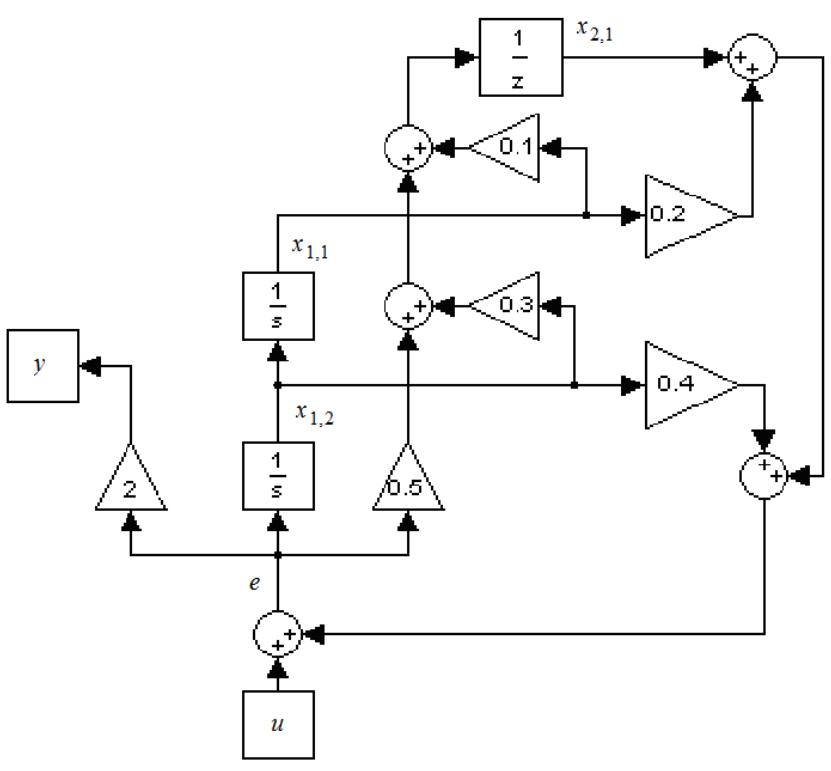

Fig. 2. State space diagram for transfer function (16)

Step 3. Using state variable diagram we can write the following equations:

$$
\begin{aligned}
& \dot{x}_{1,1}(t, i)=x_{1,2}(t, i), \\
& \dot{x}_{1,2}(t, i)=0.2 x_{1,1}(t, i)+0.4 x_{1,2}(t, i)+x_{2,1}(t, i)+u(t, i), \\
& x_{2,1}(t, i+1)=0.2 x_{1,1}(t, i)+0.5 x_{1,2}(t, i) \\
& \quad+0.5 x_{2,1}(t, i)+0.5 u(t, i), \\
& y(t, i)=0.4 x_{1,1}(t, i)+0.8 x_{1,2}(t, i)+2 x_{2,1}(t, i)+2 u(t, i) .
\end{aligned}
$$

Step 4. The desired realization of (16) has the form:

$$
\begin{aligned}
& A_{11}=\left[\begin{array}{cc}
0 & 1 \\
0.2 & 0.4
\end{array}\right], \quad A_{12}=\left[\begin{array}{l}
0 \\
1
\end{array}\right], \\
& A_{21}=\left[\begin{array}{ll}
0.2 & 0.5
\end{array}\right], \quad A_{22}=[0.5], \\
& B_{1}=\left[\begin{array}{l}
0 \\
1
\end{array}\right], \quad B_{2}=[0.5], \quad C_{1}=\left[\begin{array}{ll}
0.4 & 0.8
\end{array}\right], \\
& C_{2}=[2], \quad D=[2] .
\end{aligned}
$$

Obtained realization have only nonnegative entries and its of minimal dimension.

\subsection{Case 2}

Defining:

$$
\begin{aligned}
Y= & \left(b_{n_{1}, n_{2}}+b_{n_{1}, n_{2}-1} z^{-1}+b_{n_{1}-1, n_{2}} s^{-1}\right. \\
& \left.+\ldots+b_{00} s^{-n_{1}} z^{-n_{2}}\right) U
\end{aligned}
$$

we may draw the state variable diagram shown in Fig. 3 .

Similarly as in section 3.1 as a state variable we choose the outputs of integrators $\left(\mathrm{x}_{1,1}(t, i), \mathrm{x}_{1,2}(t, i), \ldots, \mathrm{x}_{1, \mathrm{n}_{1}}(t, i)\right)$ and of delay elements $\left(\mathrm{x}_{2,1}(t, i), \mathrm{x}_{2,2}(t, i), \ldots, \mathrm{x}_{2, \mathrm{n}_{2}}(t, i)\right)$. 


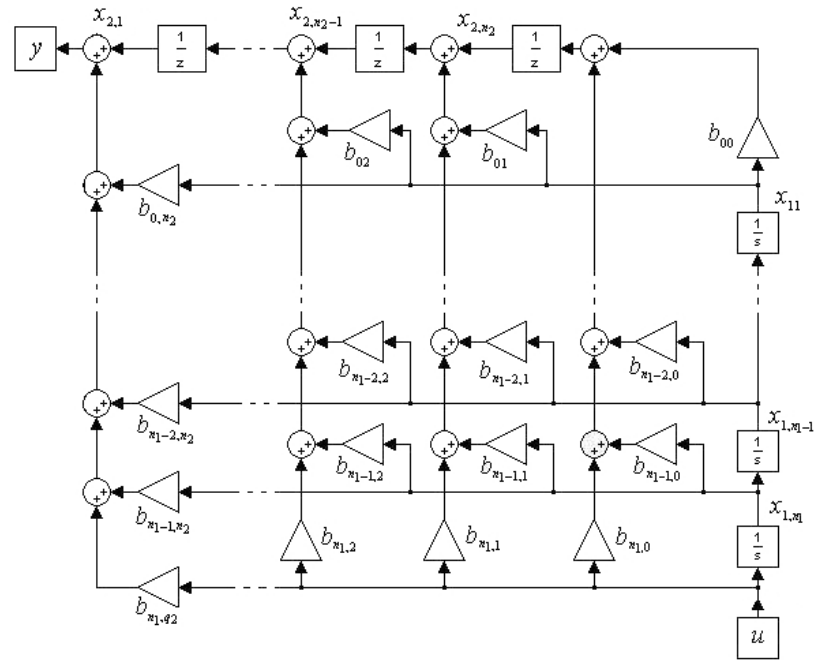

Fig. 3. State space diagram for transfer function (8) of all-zero system

Using state variable diagram (Fig. 3) we can write the following differential and difference equations:

$$
\begin{aligned}
& \dot{x}_{1,1}(t, i)=x_{1,2}(t, i) \text {, } \\
& \dot{x}_{1,2}(t, i)=x_{1,3}(t, i) \text {, } \\
& \dot{x}_{1, n_{1}-1}(t, i)=x_{1, n_{1}}(t, i) \text {, } \\
& \dot{x}_{1, n_{1}}(t, i)=u(t, i) \text {, } \\
& x_{2,1}(t, i+1)=b_{0, n_{2}-1} x_{1,1}(t, i)+b_{1, n_{2}-1} x_{1,2}(t, i)+\ldots \\
& +b_{n_{1}-1, n_{2}-1} x_{1, n_{1}}(t, i)+x_{2,2}(t, i)+b_{n_{1}, n_{2}-1} u(t, i), \\
& x_{2,2}(t, i+1)=b_{0, n_{2}-2} x_{1,1}(t, i)+b_{1, n_{2}-2} x_{1,2}(t, i)+\ldots \\
& +b_{n_{1}-1, n_{2}-2} x_{1, n_{1}}(t, i)+x_{2,3}(t, i)+b_{n_{1}, n_{2}-2} u(t, i), \\
& x_{2, n_{2}-1}(t, i+1)=b_{0,1} x_{1,1}(t, i)+b_{1,1} x_{1,2}(t, i)+\ldots \\
& +b_{n_{1}-1,1} x_{1, n_{1}}(t, i)+x_{2, n_{2}}(t, i)+b_{n_{1}, 1} u(t, i), \\
& x_{2, n_{2}}(t, i+1)=b_{0,0} x_{1,1}(t, i)+b_{1,0} x_{1,2}(t, i)+\ldots \\
& +b_{n_{1}-1,0} x_{1, n_{1}}(t, i)+b_{n_{1}, 0} u(t, i) \text {, } \\
& y(t, i)=b_{0, n_{2}} x_{1,1}(t, i)+b_{1, n_{2}} x_{1,2}(t, i)+\ldots \\
& +b_{n_{1}-1, n_{2}} x_{1, n_{1}}(t, i)+x_{2,1}(t, i)+b_{n_{1}, n_{2}} u(t, i) \text {. }
\end{aligned}
$$

Defining state vectors in the form:

$$
x_{1}(t, i)=\left[\begin{array}{c}
x_{1,1}(t, i) \\
\vdots \\
x_{1, n_{1}}(t, i)
\end{array}\right], \quad x_{2}(t, i)=\left[\begin{array}{c}
x_{2,1}(t, i) \\
\vdots \\
x_{2, n_{2}}(t, i)
\end{array}\right]
$$

we can write the equations (21) in the matrix form (14) where:

$$
A_{11}=\left[\begin{array}{ccccc}
0 & 1 & 0 & \ldots & 0 \\
0 & 0 & 1 & \ldots & 0 \\
\vdots & \vdots & \vdots & \ddots & \vdots \\
0 & 0 & 0 & \ldots & 1 \\
0 & 0 & 0 & \ldots & 0
\end{array}\right] \in \mathfrak{R}^{n_{1} \times n_{1}}, A_{12}=[0] \in \mathfrak{R}^{n_{1} \times n_{2}},
$$

$$
\begin{aligned}
A_{21} & =\left[\begin{array}{ccc}
b_{0, n_{2}-1} & \ldots & b_{n_{1}-1, n_{2}-1} \\
\vdots & \ldots & \vdots \\
b_{0,0} & \ldots & b_{n_{1}-1,0}
\end{array}\right] \in \mathfrak{R}^{n_{2} \times n_{1}}, \\
A_{22} & =\left[\begin{array}{ccccc}
0 & 1 & 0 & \ldots & 0 \\
0 & 0 & 1 & \ldots & 0 \\
\vdots & \vdots & \vdots & \ddots & \vdots \\
0 & 0 & 0 & \ldots & 1 \\
0 & 0 & 0 & \ldots & 0
\end{array}\right] \in \mathfrak{R}^{n_{2} \times n_{2}}, \\
B_{1} & =\left[\begin{array}{c}
0 \\
\vdots \\
0 \\
1
\end{array}\right] \in \mathfrak{R}^{n_{1} \times 1}, \quad B_{2}=\left[\begin{array}{c}
b_{n_{1}, n_{2}-1} \\
b_{n_{1}, n_{2}-2} \\
\vdots \\
b_{n_{1}, 0}
\end{array}\right] \in \mathfrak{R}^{n_{2} \times 1}, \\
C_{1}= & {\left[\begin{array}{lll}
b_{0, n_{2}} & \ldots & b_{n_{1}-1, n_{2}}
\end{array}\right] \in \mathfrak{R}^{1 \times n_{1}}, } \\
C_{2}= & {\left[\begin{array}{llll}
1 & 0 & \ldots & 0
\end{array}\right] \in \mathfrak{R}^{1 \times n_{2}}, \quad D=\left[b_{n_{1}, n_{2}}\right] \in \mathfrak{R}^{1 \times 1} . }
\end{aligned}
$$

Therefore, the following theorem has been proved.

Theorem 3.2. There exists a positive realization of dimension $\left(n_{1}+n_{2}\right) \times\left(n_{1}+n_{2}\right)$ if the system is all-zero and all coefficients of the nominator of transfer function (8) are nonnegative. If the assumptions of Theorem 3.2 are satisfied then a positive realization (3) of (8) can be found by the use of the following procedure.

\section{Procedure 3.2.}

Step 1. Write the transfer function (8) in the form (20).

Step 2. Using (20) draw the state variable diagram shown in Fig. 3.

Step 3. Choose the state variables and write equations (21).

Step 4. Using (21) find the desired realization (23) of transfer function (8).

Example 3.2. Find a positive realization (3) of the all-zero continuous-discrete system with proper transfer function:

$T(s, z)=6 s^{2} z+5 s^{2}+4 s z+3 s+2 z+1$

In this case $n_{1}=2$ and $n_{2}=1$.

Using Procedure 3.2 we obtain the following.

Step 1. Transfer function (24) can by written as:

$Y=\left(6+5 z^{-1}+4 s^{-1}+3 s^{-1} z^{-1}+2 s^{-2}+s^{-2} z^{-1}\right) U$

Step 2. State variable diagram has the form shown in Fig. 4.

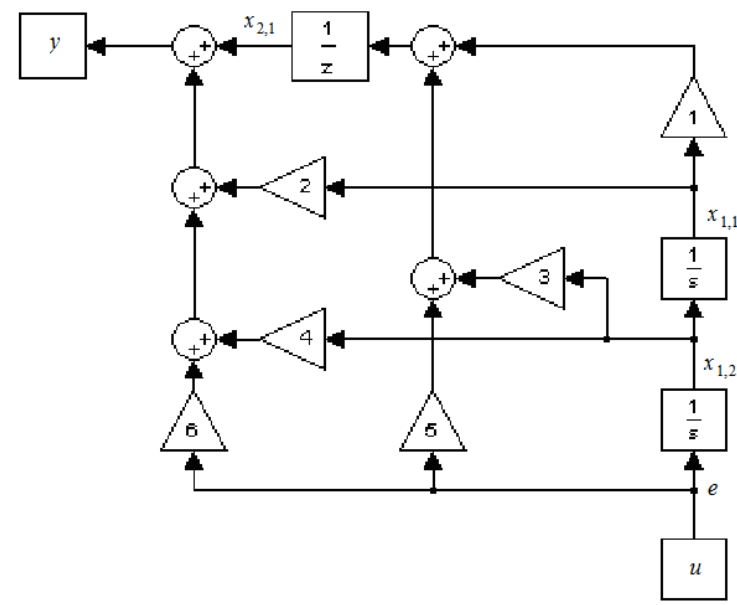

Fig. 4. State space diagram for transfer function (24) 
Step 3. Using state variable diagram we can write the following equations:

$$
\begin{aligned}
& \dot{x}_{1,1}(t, i)=x_{1,2}(t, i), \\
& \dot{x}_{1,2}(t, i)=u(t, i), \\
& x_{2,1}(t, i+1)=x_{1,1}(t, i)+3 x_{1,2}(t, i)+5 u(t, i), \\
& y(t, i)=2 x_{1,1}(t, i)+4 x_{1,2}(t, i)+x_{2,1}(t, i)+6 u(t, i) .
\end{aligned}
$$

Step 4. The desired realization of (17) has the form:

$$
\begin{aligned}
& A_{11}=\left[\begin{array}{ll}
0 & 1 \\
0 & 0
\end{array}\right], \quad A_{12}=\left[\begin{array}{l}
0 \\
0
\end{array}\right], \\
& A_{21}=\left[\begin{array}{ll}
1 & 3
\end{array}\right], \quad A_{22}=[0], \\
& B_{1}=\left[\begin{array}{l}
0 \\
1
\end{array}\right], \quad B_{2}=[5], \quad C_{1}=\left[\begin{array}{ll}
2 & 4
\end{array}\right], \\
& C_{2}=[1], \quad D=[6]
\end{aligned}
$$

Obtained realization have only nonnegative entries and its of minimal dimension.

\section{CONCLUDING REMARKS}

A method for computation of a positive minimal realization of a given proper transfer function of all-pole and all-zero continuous-discrete linear systems has been proposed. Sufficient conditions for the existence of a positive and minimal realization of a given proper transfer function have been established. Two procedures for computation of a positive minimal realizations have been proposed. The effectiveness of the procedures have been illustrated by a numerical examples. Extension of those considerations for 2D continuous-discrete linear systems described by second Fornasini-Marchesini model (Sajewski and Kaczorek, 2010) is possible.

An open problem is formulation of the necessary and sufficient conditions for the existence of solution of the positive and minimal realization problem for $2 \mathrm{D}$ continuous-discrete linear systems in the general form (Kurek, 1985).

\section{REFERENCES}

1. Antoniou G. E. (2002), Minimal state space realization for all-pole and all-zero lattice discrete 2D filters, International Journal of Systems Science, Vol. 33, No. 10, 799-803.

2. Benvenuti L., Farina L. (2004), A tutorial on the positive realization problem, IEEE Trans. on Autom. Control, Vol. 49, No. 5, 651-664.

3. Dymkov M., Gaishun I., Rogers E., Gałkowski K., Owens D. H. (2004), Control theory for a class of $2 \mathrm{D}$ continuous-discrete linear systems, Int. J. Control Vol. 77, No. 9, 847-860.

4. Farina L., Rinaldi S. (2000), Positive Linear Systems; Theory and Applications, J. Wiley, New York.

5. Kaczorek T. (1992), Linear control systems, Vol. 1, Research Studies Press J. Wiley, New York.

6. Kaczorek T. (2002), Positive 1D and 2D Systems, Springer-Verlag, London.

7. Kaczorek T. (2004), Realization problem for positive discrete-time systems with delay, System Science, Vol. 30, No. 4, 117-130.

8. Kaczorek T. (2005), Positive minimal realizations for singular discrete-time systems with delays in state and delays in control, Bull. Pol. Acad. Sci. Tech., Vol. 53, No. 3, 293-298.
9. Kaczorek T. (2006a), A realization problem for positive continuoustime linear systems with reduced numbers of delay, Int. J. Appl. Math. Comp. Sci., Vol. 16, No. 3, 325-331.

10. Kaczorek T. (2006b), Realization problem for positive multivariable discrete-time linear systems with delays in the state vector and inputs, Int. J. Appl. Math. Comp. Sci., Vol. 16, No. 2, 101-106.

11. Kaczorek T. (2007), Positive 2D hybrid linear systems, Bull. Pol. Acad. Sci. Tech., Vol. 55, No. 4,351-358.

12. Kaczorek T. (2008a), Positive fractional 2D hybrid linear systems, Bull. Pol. Acad. Tech., Vol. 56, No. 3, 273-277.

13. Kaczorek T. (2008b), Realization problem for positive 2D hybrid systems, COMPEL, Vol. 27, №. 3, 613-623.

14. Kaczorek T., Busłowicz M. (2004), Minimal realization problem for positive multivariable linear systems with delay, Int. J. Appl. Math. Comput. Sci., Vol. 14, No. 2, 181-187.

15. Kaczorek T., Marchenko V., Sajewski Ł. (2008), Solvability of 2D hybrid linear systems - comparison of the different methods, Acta Mechanica et Automatica, Vol. 2, No. 2, 59-66.

16. Kurek J. (1985), The general state-space model for a twodimensional linear digital system, IEEE Trans. on Austom. Contr., AC-30, 600-602.

17. Roesser R. B. (1975), A discrete state-space model for linear image processing, IEEE Trans. on Autom. Contr., AC-20, 1-10.

18. Sajewski Ł. (2009), Solution of 2D singular hybrid linear systems, Kybernetes, Vol. 38, No. 7/8, 2009, 1079-1092.

19. Sajewski Ł., Kaczorek T. (2009), Computation of positive realizations of singular SISO hybrid linear systems, JAMRIS, Vol. 3 , No. 4, 8-14.

20. Sajewski Ł., Kaczorek T. (2010), Computation of positive realizations of MIMO hybrid linear systems in the form of second Fornasini-Marchesini model, Archives of Control Sciences, Vol. 20, No. 3, 253-271.

21. Sun-Yuan Kung, Levy B.C., Morf M., Kailath T. (1977), New Results in 2-D Systems Theory, Part II: 2-D State-Space ModelsRealization and the Notions of Controllability, Observability and Minimality, Proc. of the IEEE, Vol. 65, No. 6, 945-961.

22. Varoufakis S. J., Paraskevopoulos P.N., Antoniou G. E. (1987), On the minimal state-space realizations of all-pole and all-zero 2-D systems, IEEE Trans. on Circ. and Sys., Vol. 34, No. 3, 289-292.

Acknowledgment: This work was supported by European Social Fund and Polish Government under scholarship no. WIEM/POKL/MD/III/2011/2 of Human Capital Programme. 